\title{
Particle Swarm Optimisation for Scheduling Electric Vehicles with Microgrids
}

\author{
Zedong Zheng \\ Centre for Computational Intelligence (CCI) \\ School of Computer Science and Informatics \\ De Montfort University \\ The Gateway, Leicester LE1 9BH, UK \\ Email: donniezzd@gmail.com
}

\author{
Shengxiang Yang \\ Centre for Computational Intelligence (CCI) \\ School of Computer Science and Informatics \\ De Montfort University \\ The Gateway, Leicester LE1 9BH, UK \\ Email: syang@dmu.ac.uk
}

\begin{abstract}
The explosion in the number of electric vehicles (EVs) has had a significant impact on the energy systems and structures of cities. Large-scale EVs inevitably increase the load on the grid, while uncoordinated vehicle to Grid (V2G) technologies pose challenges to the stability and security of the grid. This paper introduces a global intelligent method to find optimal cooperation charging/discharging strategies for EVs to minimize the operation cost. EVs aggregates co-ordinate the energy information and needs of all EVs and use real-time pricing based on micro-grid loads to influence EV charge-discharge behavior. Particle swarm optimization (PSO) is introduced to solve the EV scheduling problem. This study also discusses the negative impact on the energy system of different strategies for charging EVs. Simulation shows that this smart charging strategy and improved PSO can effectively decrease the operation cost of EVs and reduce the load for each micro-grid.
\end{abstract}

Index Terms-Optimal scheduling, Electric vehicles, Particle swarm optimisation, Microgrids, Global strategy

\section{INTRODUCTION}

Recently, electric vehicles (EVs) are rapidly increasing in number because of their significant advantages: high energy efficiency and green transportation [1]. Furthermore, the breakthrough of battery material technologies and the development of rapid charging technologies have more eased the bottleneck problems which affect the usability of EVs. However, the construction of the existing energy infrastructure is far behind the growing trend of EVs. EVs bring not only convenience and environmental protection but also treats and challenges to the energy system, such as load grows, voltage decrease, frequency concussion, network congestion, etc [2], [3]. The potential factors of EVs which affect the urban energy system are battery capacity, charging requirement, travel behavior. As EVs spread as a means of transportation, the impact will be felt across different power networks.

On the other hand, the management of charging of EVs is a very complex problem with unpredictable dynamics and volatility [4]. Besides, different vehicle to grid technologies which achieve the two-way interaction between the energy system and EVs also make the issue for connecting EVs with a grid more complicate [5], [6]. Coordinated charging can effectively improve the utilization rate of energy, while irregular charging aggravates the system load of the energy system.
Similarly, coordinated discharge can effectively reduce the load demand of the energy system, while disorder discharge can impact the stability and security of the power network [7]. Therefore, it is more important to study economic and effective scheduling strategies to manage charging and discharging behaviour of EVs to enhance the potential beneficial effects, such as flattening the load curve [8], reducing the system cost [9] and operation losses [10].

The scheduling of EVs should first meet their transportation demands, then enable their reasonable interaction with the grid. Considering these factors, for specific application scenarios, an appropriate scheduling strategy and an efficient optimization solution are essential. At present, a lot of work has been done to study the optimal scheduling strategy of EVs. Here, we briefly summarize it from the perspective of models and algorithms.

From the model point of view, some specific researches of EV scheduling have been developed for EV coordination management. Tushar et al. [11]proposed a central joint control scheduling method based on residential MG. The technique focuses on the surplus power of EVs within the local area to enhance the reliability and stability of the energy system by coordinating with household appliances. Thomas et al. [12] investigated the impact of the randomness of electric cars connected to a smart office building MG on a power system. In this research, the bi-directional energy trading capability of EVs is considered within working time. Mavrovouniotis et al. [13] considered the scheduling problem for charging EVs within a charging station which is designed to be installed in a parking area. The goal of management is to coordinate the charging process of EVs parked in the parking lot to reduce potential damage to the charging station. Shaaban et al. [14] proposed an on-line coordination strategy for EVs charging control in a smart distribution network. The goal of this research is to maximize the owners satisfaction and minimize system operation costs without exceeding the limit. Due to the uncertainty of EVs travel behavior, it is observed that most of the researches focus on how to manage the EVs which are connected in a specific energy network. However, in practice, the distance by using EVs as daily travel is more than the range of two energy grids. For example, commuter cars 
will connect the energy grid in the home area and the working area separately in a typical working day. Therefore, the overall impact of charging and discharging of EVs on different MGs should also be concerned.

So far, most of the strategies and modelling for coordinated management of EV charge and discharge have generally been based on linear modelling, especially mixed integer linear models [11], [12]. Some scholars have also modelled the scheduling problem as a convex optimisation problem through the relationship between EVs and the power grid. They prefer to solve these problems quickly by using business optimisation tools, such as GAMS [15], CVX [16], and CPLEX [17]. On the other hand, some conventional mathematical optimisation methods are also used to solve the scheduling problem of EVs [18]. However, when the scale of EVs increases, the temporal and spatial dimensions increase, or the model complexity increases, these methods will get into trouble. Therefore, now some intelligent optimisation algorithms, such as particle swarm optimisation (PSO) [19], differential evolution (DE), genetic algorithm (GA) [20], and ant colony optimisation (ACO) [13], and so on, are also applied to the management of EVs. It has been verified by a large number of scholars that these new intelligent algorithms are more advantageous than the traditional methods for solving large-scale and complex problems [21]. However, it should be noted that any solution method should be chosen, adjusted and improved according to the specific situation of the solution in practice.

Based on the analyses above, a global dispatching model of EVs is proposed by considering the two-way influence between EVs and MGs within an urban area in this research. The main aim of the problem is to reduce the impact on the power system while meeting the transportation and economic needs of EVs. PSO is used as a suitable method to solve the problem. And some parameters are adjusted according to the practice application. The simulation results also show that the implementation of a global optimization scheduling strategy has positive effects on both EVs and MGs.

The remainder of this paper is organized as follows. In Section 2, the system framework of the scheduling problem is introduced, and the mathematical model of an optimization problem is also proposed. In Section 3, the optimal scheduling strategy is presented in detail. In Section 4, a PSO algorithm with some improvement according to the proposed approach is introduced to solve the optimization object. In Section 5, simulation based on two MGs is presented to investigate the performance of the proposed charging strategy. Finally, the conclusion will be presented in Section 6 .

\section{PROBLEM DESCRIPTION AND SCHEDULING MODEL}

An urban energy network usually is made up of various communities and economic areas, such as residential, business, factory and agricultural areas. As an efficient method of energy management, the time-of-use and tiered pricing result in electric price differences between different places and time periods.

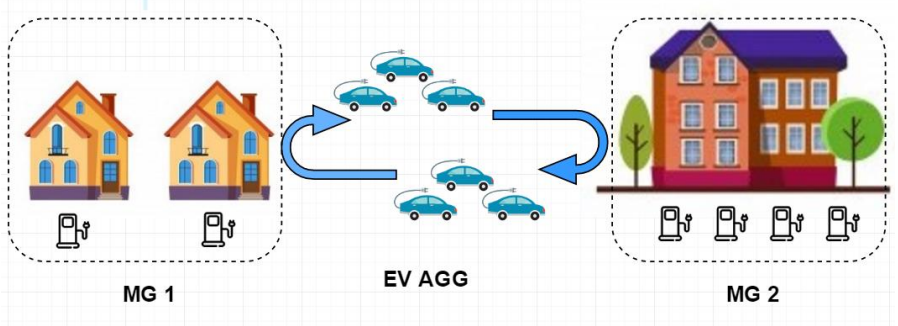

Fig. 1. Optimized scheduling framework.

In view of the above situation, the optimal scheduling of EVs should be considered from time and space as a whole. As Fig. 1 shows, the energy framework consists of two different types of MGs in this paper. A certain number of EVs are used for transportation between the two energy systems. Therefore, EVs will travel between two MGs and stay on a certain period on both areas during a typical working day. From a basic travel perspective, the optimal dispatch process should consider the car owner schedule and requirements first. For ease of operation, EV aggregates are introduced into the management framework to coordinate the energy interaction.

\section{A. Electric Vehicles}

The impact of EVs on the energy system mainly depends on the travel patterns, energy demand and battery characteristics. Traffic patterns determine the travel status of an EV, the energy consumption speed of energy, the connecting and off-line time. Energy demand depends on the level of energy required by the owner while the vehicle is leaving. The charging and discharging power and rate, overall capacity is up to the battery characteristics. In particular, the travel patterns and energy demand of EVs are mainly concerned.

For each EV, the status of charge (SOC) is the relative energy level:

$$
S O C=\frac{E}{E_{\text {cap }}} \cdot 100 \%
$$

where $E$ is the existing energy of the battery, $E_{c a p}$ is the rated capacity of the battery which comes from the EV manufacturer. The $S O C$ is totally up to the status of EV and charging/discharging. When an EV has been disconnected from the grid, the battery's self-discharge and the energy consumption of travel are the main reasons for the SOC varies. When the EV is connected to the power grid, the SOC change is determined by the charging, discharging, or idle mode of the EV.

In this study, a day-ahead energy scheduling strategy will be implemented, and the time will be divided into 24 intervals which means the interval length is an hour per term. Set $t=$ $1,2,3 \ldots T$, where $t$ is the index for the time slot contained in one day duration, and $T=24$. Assume $g=1,2, \ldots, G$ is the index for the period when EVs are connected with one $\mathrm{MG}$ in the one-time slot. $i$ represents which MG is connected. The number of EVs is $N$, so the index of EVs can be expressed as $n=1,2, \ldots, N$. From an operational point of view, when the 
vehicle arrives at an $\mathrm{MG}$, it can be regarded as connected to the system, and the time of departure can be considered as off the grid. Assuming that the driving behavior of all EVs can be predicted, which means the following information is available for each EV: 1) the initial $\left.S O C_{i n i} ; 2\right)$ the travel distance $D_{n}$ between two MGs for each EV; 3) The travel plan for each $\mathrm{EV}$, including the arrival time $t_{a r r}$, departure time $t_{\text {lea }}$, and travel time $t_{\text {tra }}$. Therefore, the whole scheduling period can also be expressed as follows:

$$
T=\left[t_{a r r, 1} . . t_{l e a, 1}, t_{t r a} . . t_{a r r, 2} . . t_{l e a 2}, t_{t r a} . . t_{a r r, G} . . t_{l e a, G}, \ldots T\right]
$$

- When the $n$th $\mathrm{EV}$ is traveling:

$$
E_{n, t}=E_{n, t-1}-\frac{D_{n}}{E_{c a p}} \cdot D_{n_{r} a} \triangle t
$$

Here $D_{n_{r} a}$ denotes the rated travel distance of the $n$th EV. $E_{n, t}$ indicates the energy value of the $n$th $\mathrm{EV}$ at time $t$.

- When the $n$th $\mathrm{EV}$ is connected with the MG:

$$
E_{n, t}=E_{n, t-1, g}+E_{n, t-1, g}^{o b} \Delta t
$$

Here, $E_{n, t-1, g}^{o b}$ refers to the amount of energy that can be obtained from the $n$th $\mathrm{EV}$ in the $(t-1)$ th time with the $g$ th MG. As mentioned earlier:

- When the EV is charged, the charging power $P_{n, t-1, g}>$ 0 , and $E_{n, t-1, g}^{o b}=\frac{P_{n, t-1, g}}{\eta_{c h}}$.

- When EV is discharged, the charging power $P_{n, t-1, g}<$ 0 , and $E_{n, t-1, g}^{o b}=\frac{P_{n, t-1, g}}{\eta_{d c h}}$;

- While $P_{n, t-1, g}=0, \mathrm{EV}$ is idle, and $E_{n, t-1, g}^{o b}=0$.

Here, $\eta_{c h}$ and $\eta_{d c h}$ are the efficiency of charge and discharge respectively.

\section{B. Objective Function and Constraints}

Economic factors are one of the main factors that affect the participation of EV owners in the optimal management. Therefore, the optimal scheduling objective selected in this paper is to minimize the daily operation cost of the EV aggregation, and function as follows:

$$
\operatorname{Min} F=\sum_{t}^{T} \sum_{n}^{N} P_{n, t} \cdot C_{t}^{\text {price }}
$$

The decision variable $P_{n, t}$ is the charging power of each $\mathrm{EV}$ at each time slot. The price variable $C_{t}^{\text {price }}$ depends on which MG the $n$th EV is charged at the moment $t$. Considering the volatility and randomness of the energy system, the price strategy adopted in this paper is different from the price strategy immobilized one day ago. This pricing strategy will vary strictly according to the timing and load demand of the energy system. The price function is given in Eq. (4), and adopted to model the dynamic charging/discharging price [17].

$$
C_{\text {price }, t}=k_{1}+\left(s_{f a} \cdot\left(P_{t, g}^{\text {load }}+\sum_{n}^{N} P_{n, t, g}\right)\right) \cdot k_{2}
$$

where $k_{1}, s_{f a}, k_{2}$ are the price coefficients, and $P_{t, g}^{\text {load }}$ is the total predicted load of the $g$ th MG. Then, combining Eq. (5) with Eq. (6), we can get the final cost function (7), which is in a non-linear form.

$\operatorname{Min} F=\sum_{t}^{T} \sum_{n}^{N} \sum_{g}^{G}\left(P_{n, t, g} \cdot\left(k_{1}+s_{f a} \cdot\left(P_{\text {load }, t, g}+\sum_{n}^{N} P_{n, t, g}\right) \cdot k_{2}\right)\right)$

Next, we describe the constraints for the optimization problem. For protecting the battery life, the SOC level of each EV should be kept within the safe range which is recommended by the manufacturer. So, the first constraint is given as follows:

$$
E_{n}^{\min } \leq E_{n, t} \leq E_{n}^{\max }, \forall n, t
$$

where $E_{n}^{\min }$ and $E_{n}^{\max }$ denote the minimum and maximum values for $\mathrm{EV} n$.

When EVs are connected to the grid for charging or discharging, the power of charging and discharging is limited, and it can be considered that EVs cannot charge and discharge at the same time in the same scheduling time slot. It also means that when EVs are disconnected with the MG or within the travel time slot, the charging power is zero.

$$
-\left|P_{n}^{D E \max }\right| \leq P_{n, t} \leq\left|P_{n}^{C H \max }\right|
$$

where $P_{n}^{D E \max }$ and $P_{n}^{C H \max }$ represent the maximum discharging and charging limits, respectively.

At the same time, each vehicle has its own energy needs based on its travel needs, which should be guaranteed when they leave the MG.

$$
E_{n, g, t_{l e a}} \geq E_{n, g}^{r e q} \geq E_{n}^{t r a}
$$

where $E_{n, m_{i}, t_{l e a}}$ indicates the energy level when the $n$th EV leaves the $i$ th $\mathrm{MG}$, and $E_{n, g}^{r e q}$ denotes the requirement level of the owner when the EV is out from the $i$ th MG. Both of them should be greater than or at least equal to the energy needed for the next trip. These values should also not exceed the manufacturer's battery safety limits with Eq. (8).

\section{Optimization METHOD}

\section{A. Particle swarm optimization (PSO)}

PSO is a heuristic algorithm based on a stochastic optimization of swarm intelligence. The basic concept was inspired by the analysis and study of the foraging behavior of birds, which was proposed by Eberhart and Kennedy in 1995 [22]. Because of its simple operation and fast convergence speed, PSO has been widely used in vehicle scheduling, energy management and other fields. However, PSO has some problems in practical applications, such as premature convergence, dimension disaster, and easy to fall into local optima. So, the algorithm should be adjusted and improved according to the actual optimization problem.

In PSO, each particle can be regarded as a searching individual in an $\mathrm{N}$-dimensional real space, the current position of the particle is a candidate solution of the corresponding optimization problem, and the flight process of the particle is the search process of the individual. The flight speed and direction of particles are dynamically adjusted according to the optimal historical position of particles and the optimal 
historical position of the population. Therefore, each particle has two properties: its velocity, which represents how fast it moves, and location. The optimal solution of individual search for each particle is called individual extremum, and the optimal individual extremum in the swarm is regarded as the current global optimal solution. The particles will update their velocity and location iteration by iteration until the termination condition is satisfied.

The velocity and location update formulas of the standard PSO are expressed as follows:

$$
V_{i, d+1}=\varpi \cdot V_{i, d}+c_{1} \cdot \operatorname{rand} \cdot\left(P_{i, d}-X_{i, d}\right)+c_{2} \cdot \operatorname{rand} \cdot\left(P_{g, d}-X_{i, d}\right)
$$

$$
X_{i, d+1}=X_{i, d}+V_{i, d+1}
$$

where $V_{i, d}$ refers to the $i$ th particle velocity for $d$ th iteration in the $\mathrm{N}$-dimensional search space, $X_{i, d}$ indicates $i$ th particle position for the $d$ th iteration, and similarly, $P_{i, d}$ is the best position of an individual particle, $P_{g, d}$ is the optimal global solution in the same iteration and dimension. $\varpi$ is called the inertia weight and by adjusting it, the global and local optimization performance of PSO can be adjusted accordingly. rand is a random parameter with a value range within $[0,1]$. $c_{1}$ and $c_{2}$ denote learning factors, of which the former is the individual learning factor while the latter is the social learning factor, and they usually are set as a constant.

From Eq. (12), the position of the particle at the next moment is determined by the current position and velocity, while the current velocity is determined by the original velocity, individual extremum and global extremum.

\section{B. Solving problem}

1) Constraint pre-processing: Considering the transition of EV among different MGs, the proposed scheduling strategy can be regarded as a multi-period optimization problem. Due to the strong time coupling between the energy level of EVs, the value of $P_{n, t}$ depends on $P_{n, t-1}$ with Eqs. (4), (8), (9) and (10). If the range of variables and constraints are considered, it would be difficult to solve the problem directly. So, the constraints will be compressed first in this research.

According to Eq. (10), the SOC of each EV, while disconnected with each $\mathrm{MG}$, should be greater than or equal to the predetermined requirement value of the owner, and also more than the minimum limit of the SOC. To ensure that the constraint is satisfied, the initial energy from the last previous period plus the maximum charging power at least should be more than the energy required at this moment. In this way, the SOC minimum level at each previous moment can be updated according to the final energy requirement level.

The arrival time $t_{a r r}$, departure time $t_{l e a}$, maximum charging rate $P_{n}^{C H \max }$, minimum battery level $E_{n}^{\min }$, and the charging level $E_{n, r e q}$ expected for each EV can be obtained trough prediction and collection first, while an EV is connected to one MG. Then, the new minimum power value $E_{n, t}^{N e w M i n}$ which the EV should reach can be deduced by calculation with Algorithm 1 at each moment.

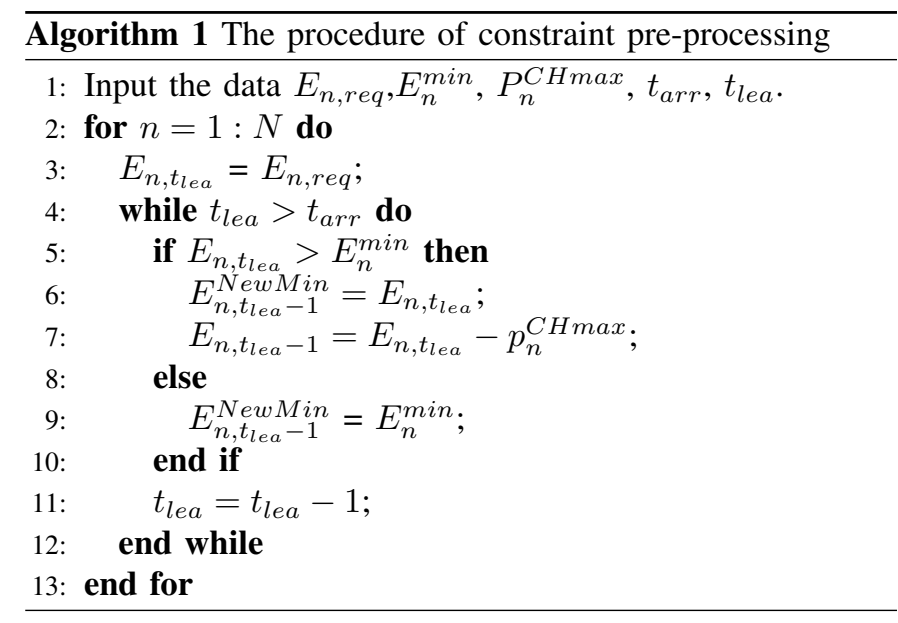

2) Algorithm adjustment: Like most stochastic algorithms, PSO also needs to balance the diversity of population and the convergence speed of the algorithm in the optimization process, so as to achieve the best performance of the algorithm. According to the function of inertial weight, a linear decreasing function (see Eq. (13)) is used to replace the original fixed weight. This method can enlarge the global search scope at the initial stage and improve the convergence accuracy of the PSO at the later stage.

$$
\varpi=\left(\varpi_{\max }-\varpi_{\min }\right) \cdot(g e r-t)+\varpi_{\min },
$$

where $\varpi_{\min }$ and $\varpi_{\max }$ are the upper and lower limits of the values of $\varpi$, and ger is the total number of algorithm iterations, and $t$ is the count of current iterations.

A variation rule is introduced to increase the diversity of PSO. In each generation of evolution, ten percent of the population size was randomly selected for mutation, and variation equation as follows:

$$
\begin{aligned}
& \gamma_{v}=0.1 \cdot(1+4 \cdot \cos (t * P i / g e r)) \\
& X_{i, d}=\left(X_{i, d}^{\max }-X_{i, d}^{\min }\right) \cdot \gamma_{v}+X_{i, d},
\end{aligned}
$$

where $\gamma_{v}$ is mutation factor, and $X_{i, d}^{\max }, X_{i, d}^{\min }$ are the upper and lower limits of the $d$ th dimension of $i$ th particle.

The proposed PSO algorithm for solving the EV scheduling problem with MGs is shown in Fig. 2

\section{Simulation STUdy}

In this section, optimization strategies and performance of optimization algorithms are discussed in the form of case studies through simulation experiments.

\section{A. Parameters setting}

Two types of typical MGs are selected to set an experimental environment of simulation, namely office MG (MG1) and residential MG (MG2). The basic load for each moment of the two MGs is shown in Fig. 3. For the simulation time, we take one day as the total optimal scheduling time, and each hour is a time interval, that is, 24 intervals in total. The commute time for an EV is usually connected to the office at $9-18$, and to the home at $20-8$. With respect to the randomness 


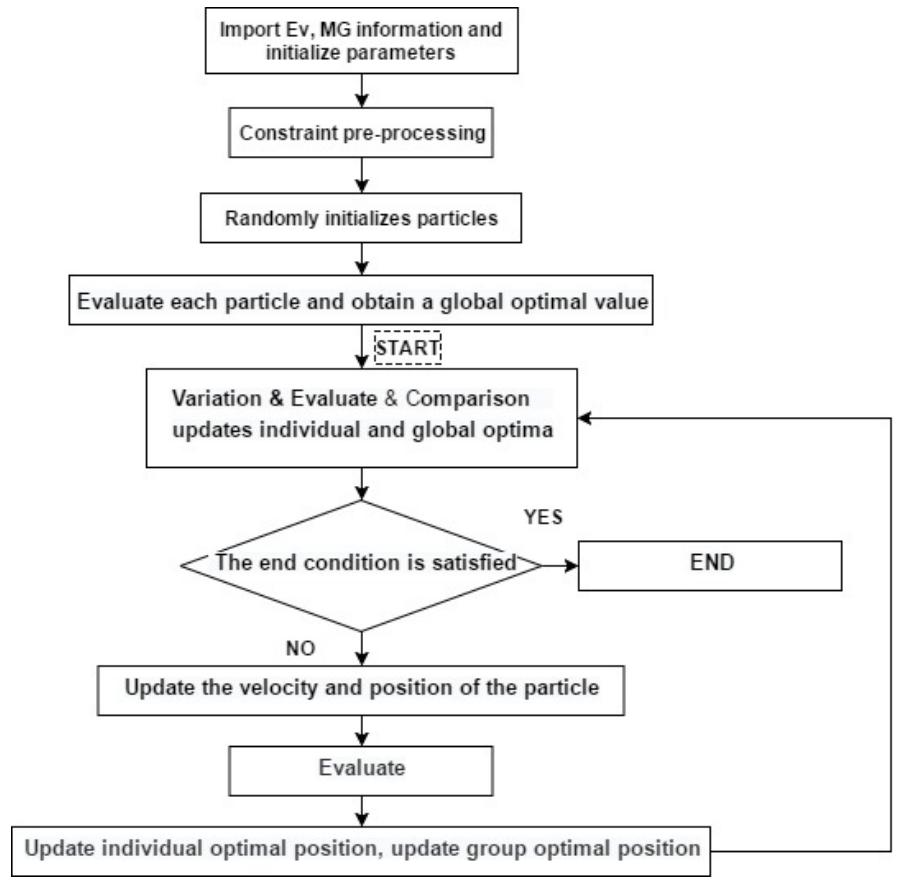

Fig. 2. The proposed PSO algorithm.

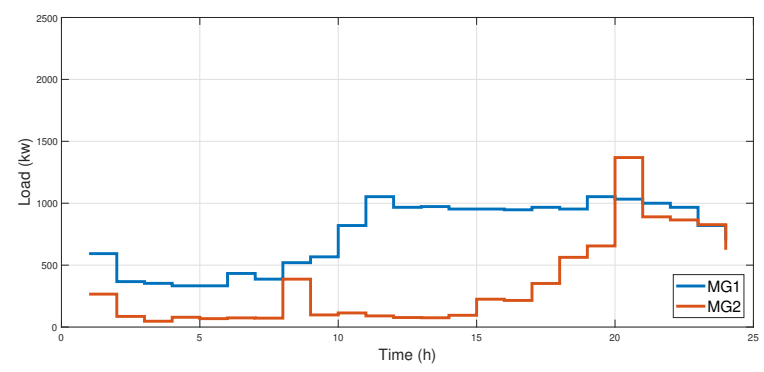

Fig. 3. The predicted load of MGs.

in the behavior of EVs, Monte Carlo simulation is used to generate the travel distance and initial battery level for each EV. Relevant data of the simulation are summarized in Table I. To facilitate understanding and comparison of the results, some assumption should be declared before simulation.

- All EVs participate in the coordination through EV aggregates, scheduling in response to price strategies.

- Other energy losses of EVs are ignored, and only the energy consumed by the trips is considered.

\section{B. Simulation results}

The simulation was implemented by MATLAB R2017a on a laptop with i7 CPU and 8G memory. The simulation is divided into five cases. For comparison purposes, the final off-grid energy requirements of the first four cases were set to the maximum. The fifth case is used to discuss the effects of different energy requirements. The description of the cases and the simulation results of each case are provided below.
TABLE I

PARAMETER SETTINGS

\begin{tabular}{|l|l|}
\hline \multicolumn{2}{|c|}{ Model parameters } \\
\hline EV numbers & 100 \\
\hline Battery capacity & $E_{c a p}=40(\mathrm{kw})$ \\
\hline Maximum/Minimum level of battery & $E_{n}^{\text {min }}=4, E_{n}^{\text {max }}=40(\mathrm{kw})$ \\
\hline Maximum charging/discharging rate & $P_{n}^{D E m a x}=P_{n}^{C H m a x}=7(\mathrm{kw})$ \\
\hline Efficient of charging/discharging & $\eta_{\mathrm{ch}}=\eta_{\mathrm{ch}}=100 \%$ \\
\hline Travel energy level distribution range & $E_{n}^{\text {tra }} \in[8 \%, 35 \%] \cdot E_{\text {cap }}$ \\
\hline Initial energy level distribution range & $E_{n}^{\text {ini }} \in[40 \%, 65 \%] \cdot E_{c a p}$ \\
\hline \multicolumn{2}{|c|}{ PSO setting } \\
\hline Population size & 50 \\
\hline Inertial weight & $\varpi_{\min }=0.2, \varpi_{\max }=0.8$ \\
\hline Learning factors & $c_{1}=c_{2}=0.5$ \\
\hline Iterations & 100 \\
\hline
\end{tabular}

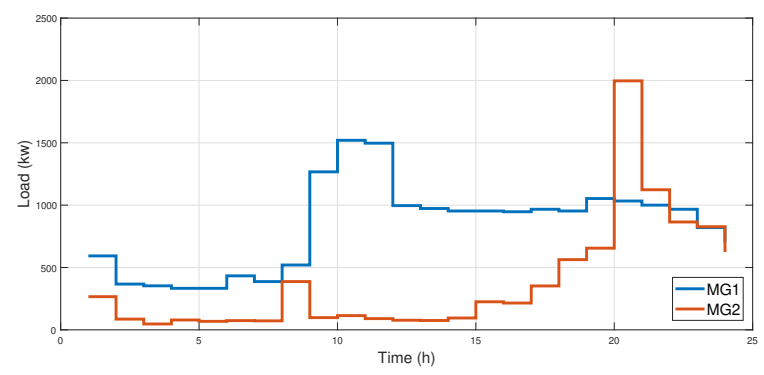

Fig. 4. Load curve for Case 1: General charging condition without optimization.

Case 1: General charging condition without optimization: Case 1 is the base for the following other cases. In this case, all EVs are not subject to optimal scheduling management, i.e., first reach first charge, which means all EVs will be directly charged while reaching any MG. Once reaching the maximum value of SOC, the process will be finished. Therefore, EVs will not generate revenue from selling energy to the grid, and will not be charged on the moderate price. On the other hand, MG can not dispatch EVs through dynamic price to reduce the impact of EVs penetration. As a result, the operation cost of EVs aggregation is $\$ 360.1449$, and the load curve is as shown in Fig. 4. It can be seen from the results that the arrival of EVs at the peak time of MGs will only worsen the situation of the whole system.

Case 2: Optimizing charging in both MGs: In Case 2, EVs are set to charge only, but not to communicate bidirectionally with the grid via V2G. By implementing the global optimal scheduling strategy, EVs can only find a suitable charging period in response to the market price, thus reducing the charging cost. In this situation, EVs cannot get the benefit by feeding the electricity back to the grid. Similarly, the grid cannot reduce the load by absorbing the extra power from EVs, only shifting the load to reduce the system's pressure while EVs are connected. The total cost of EVs in this case is $\$ 281.1522$, and the load curve is as shown in Fig. 5.

Case 3: Optimization charging and discharging for each $M G$ : In this case, the scenario that EVs can only access a single MG is optimized, but the global optimization of 


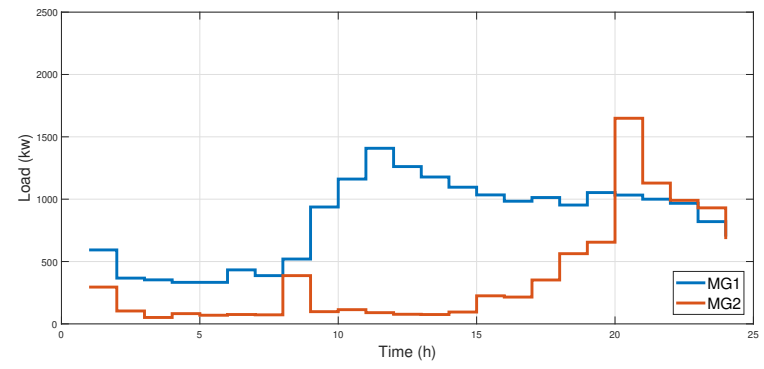

Fig. 5. Load curve for Case 2: Optimising charging in both MGs.

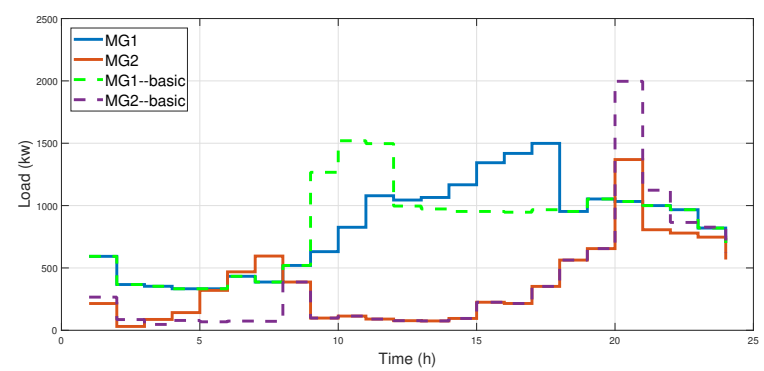

Fig. 6. Load curve for Case 3: Optimization charging and discharging for each MG.

EVs connecting to two MGs is not considered. Therefore, the results of connecting EVs to residential MG and office MG will be analyzed, respectively. The benefits obtaining of EVs can only come from the optimized portion, and the cost remains high for an unscheduled connection. The effect of the result is also for MGs. The simulation results show that when only the residential MG2 is optimized, the total operation cost is \$277.1427, and only optimal with MG1 is \$331.2217. In addition, it can be clearly seen from the change of load curve in Fig. 6 that if only a single scenario is optimized without considering the daily travel of EVs, the impact of EVs on the power grid will still exist in the non-optimized portion.

Case 4: Optimization charging and discharging for both MGs: In this case, the proposed scheduling model is applied to the optimization process, which means that EVs will be optimal charged and discharged on both MGs. In this situation, the energy requirement of EVs while disconnected to each MG is the same as the above two cases and set to the maximum of the SOC. Within the optimization time of a day, EVs can get optimized scheduling no matter which MG they are connected to. They can not only charge at the appropriate price but also sell back the excess power to the grid to earn profits. Therefore, the total cost of this case is minimal compared to the scenario above, and the final operation cost is $\$ 257.0839$. At the same time, as can be seen from the load curve, the travel of EVs does not bring more severe burden to both grids.

Case 5: Further investigations: It is worth noting that in the simulation, we found that if the required energy level is set reasonably instead of the firm level, the performance will be better. For example, based on the base loads of two MGs

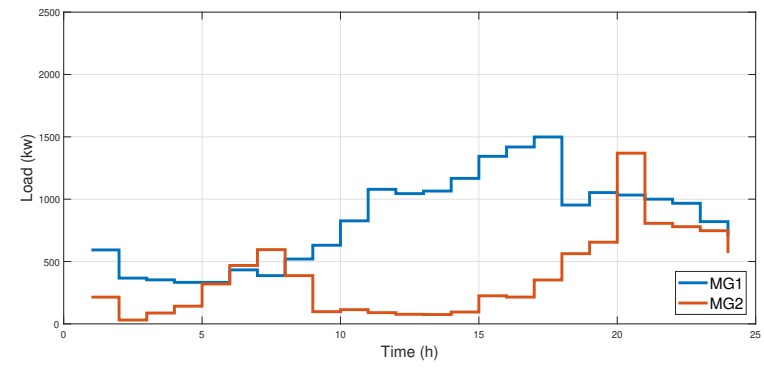

Fig. 7. Load curve for Case 4: Optimization charging and discharging for both MGs.

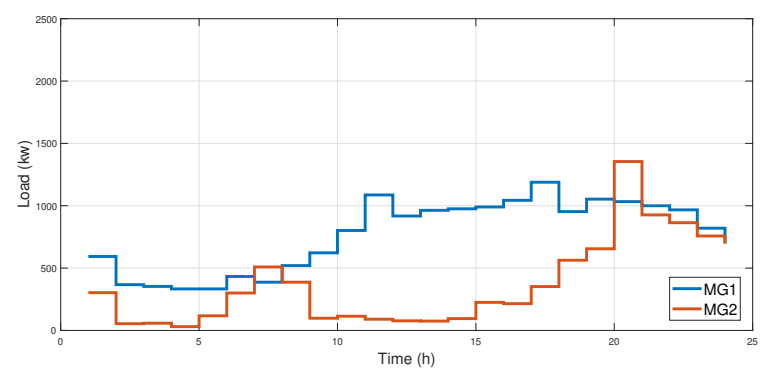

Fig. 8. Load curve for Case 5: Different levels of requirements.

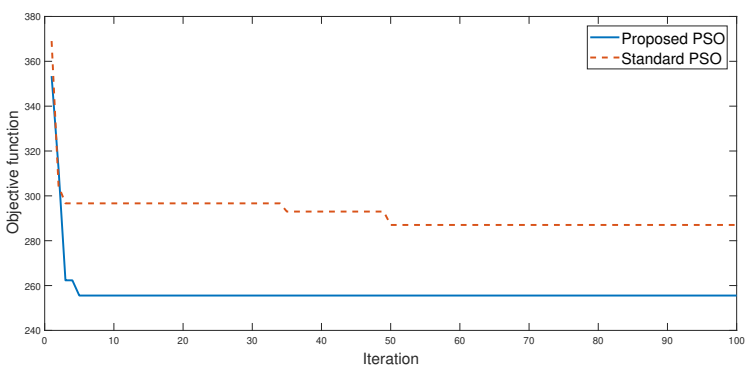

Fig. 9. Comparison of the proposed and standard PSO algorithms.

above, the level of requirements can be set as the maximum while leaving the MG2 (residential MG), and 50\% capacity while disconnecting with MG1 (Office MG). Then, the overall operation cost will be significantly reduced to $\$ 158.6271$. And the load of the MG as shown in Fig. 8 will be smoother. However, this method requires the car owner is clear about the travel plan, and coordinated with the load fluctuation of the power system.

Through all simulation results above, it can be found that the proposed optimal scheduling strategy can effectively reduce the operation cost of EVs aggregation without bringing too much impact to the power grid and the whole strategy can also be adjusted according to the actual application scenario to enhance the effect.

In addition, in order to evaluate the performance difference between the proposed PSO algorithm and the standard PSO algorithm, these two algorithms are run 30 times and the best performance of each algorithm is shown in Fig. 9. Under the 
same conditions which set the number of iterations to 100 and the population size to 100 , the accuracy of the proposed PSO is better than the standard PSO. Therefore, it can be shown that the proposed PSO algorithm is also well adapted to the solution of this problem, and can quickly converge and obtain the optimal solution.

\section{Conclusions}

The optimal management and scheduling of EVs in cities should not only consider the impact on a regional energy system but should be comprehensively evaluated in combination with the travel mode of EVs. At the same time, the optimal dispatching of EVs should not only meet the benefits of an EV owner but also can actively explore the role in assisting the energy system.

This article considers optimal scheduling schemes for EVs commuting within an urban area in one day. The whole dispatching strategy fully takes into account the normal travel behaviors of EVs in daily operation. The proposed scheduling strategy positively reduces the energy cost of EVs, and also plays a positive role in the energy system. PSO is used to solve the scheduling problem, and some adaption is made according to the specific situation of the problem. The strategy and algorithm are verified in the simulation results.

EVs will play a very important role in smart cities in the future, whether it is the travel demand at the traffic level or the auxiliary role at the power grid level. At the same time, we can also see the great challenges brought by the diversity and the increasing number of EVs. In the future, we will not only further explore the efficient and practical optimal scheduling strategy, but also study the dimension disaster brought by large-scale EVs. How to keep pace with the problems and provide more suitable solutions also needs to be paid more attention.

\section{REFERENCES}

[1] M. Sorrentino, G. Rizzo, and L. Sorrentino, "A study aimed at assessing the potential impact of vehicle electrification on grid infrastructure and road-traffic green house emissions," Applied Energy, vol. 120, pp. 3140, May 2014.

[2] S. Deilami, A. S. Masoum, P. S. Moses, and M. A. Masoum, "Realtime coordination of plug-in electric vehicle charging in smart grids to minimize power losses and improve voltage profile," IEEE Transactions on Smart Grid, vol. 2, pp. 456-467, Sep 2011.

[3] S. Aghajani and M. Kalantar, "A cooperative game theoretic analysis of electric vehicles parking lot in smart grid," Energy, vol. 137, pp. 129139, 2017.

[4] A. Weis, P. Jaramillo, and J. Michalek, "Estimating the potential of controlled plug-in hybrid electric vehicle charging to reduce operational and capacity expansion costs for electric power systems with high wind penetration," Applied Energy, vol. 115, pp. 190-204, Feb 2014.

[5] C. Fernandes, P. Frías, and J. M. Latorre, "Impact of vehicle-to-grid on power system operation costs: The Spanish case study," Applied Energy, vol. 96, pp. 194-202, 2012

[6] D. Dallinger, S. Gerda, and M. Wietschel, "Integration of intermittent renewable power supply using grid-connected vehicles - A 2030 case study for California and Germany," Applied Energy, vol. 104, pp. 666682, 2013.

[7] J. Su, T. T. Lie, and R. Zamora, "Modelling of large-scale electric vehicles charging demand: A New Zealand case study," Electric Power Systems Research, vol. 167, pp. 171-182, Feb 2019.
[8] D. Wu, D. C. Aliprantis, and L. Ying, "Load scheduling and dispatch for aggregators of plug-in electric vehicles," IEEE Transactions on Smart Grid, vol. 3, pp. 368-376, Mar 2012.

[9] Z. Liu, Q. Wu, S. Huang, L. Wang, M. Shahidehpour, and Y. Xue, "Optimal Day-Ahead Charging Scheduling of Electric Vehicles Through an Aggregative Game Model," IEEE Transactions on Smart Grid, vol. 9, pp. 5173-5184, Sep 2018.

[10] J. Tan and L. Wang, "Integration of plug-in hybrid electric vehicles into residential distribution grid based on two-layer intelligent optimization," IEEE Transactions on Smart Grid, vol. 5, no. 4, pp. 1774-1784, 2014.

[11] M. H. K. Tushar, C. Assi, M. Maier, and M. F. Uddin, "Smart microgrids: Optimal joint scheduling for electric vehicles and home appliances," IEEE Transactions on Smart Grid, vol. 5, pp. 239-250, Jan 2014.

[12] D. Thomas, O. Deblecker, and C. S. Ioakimidis, "Optimal operation of an energy management system for a grid-connected smart building considering photovoltaics' uncertainty and stochastic electric vehicles' driving schedule," Applied Energy, vol. 210, pp. 1188-1206, Jan 2018.

[13] M. Mavrovouniotis, G. Ellinas, and M. Polycarpou, "Electric Vehicle Charging Scheduling Using Ant Colony System," in Proceedings of the 2019 IEEE Congress on Evolutionary Computation (IEE CEC 2019), pp. 2581-2588, June 2019 .

[14] M. F. Shaaban, M. Ismail, E. F. El-Saadany, and W. Zhuang, "Real-time PEV charging/discharging coordination in smart distribution systems," IEEE Transactions on Smart Grid, vol. 5, no. 4, pp. 1797-1807, 2014.

[15] X. Zhang and Q. C. Zhong, "Improved Adaptive-Series-VirtualImpedance Control Incorporating Minimum Ripple Point Tracking for Load Converters in DC Systems," IEEE Transactions on Power Electronics, vol. 31, pp. 8088-8095, Dec 2016.

[16] Y. He, B. Venkatesh, and L. Guan, "Optimal scheduling for charging and discharging of electric vehicles," IEEE Transactions on Smart Grid, vol. 3, no. 3, pp. 1095-1105, 2012.

[17] K. M. Tsui and S. C. Chan, "Demand response optimization for smart home scheduling under real-time pricing," IEEE Transactions on Smart Grid, vol. 3, no. 4, pp. 1812-1821, 2012.

[18] Z. Tan, P. Yang, and A. Nehorai, "An optimal and distributed demand response strategy with electric vehicles in the smart grid," IEEE Transactions on Smart Grid, vol. 5, pp. 861-869, March 2014.

[19] C. H. Dharmakeerthi, N. Mithulananthan, and T. K. Saha, "Planning of electric vehicle charging infrastructure," in IEEE Power and Energy Society General Meeting, 2013.

[20] J. García-Álvarez, M. A. González, and C. R. Vela, "A genetic algorithm for scheduling electric vehicle charging," in Proceedings of the 2015 Genetic and Evolutionary Computation Conference, pp. 393-400, Association for Computing Machinery, Inc, Jul 2015.

[21] J. Soares, H. Morais, T. Sousa, Z. Vale, and P. Faria, "Day-ahead resource scheduling including demand response for electric vehicles," IEEE Transactions on Smart Grid, vol. 4, no. 1, pp. 596-605, 2013.

[22] Y. Shi and R. Eberhart, "Modified particle swarm optimizer," in Proceedings of the IEEE Conference on Evolutionary Computation, pp. 69-73, 1998. 